\title{
Benzodiazepine prescribing patterns and deaths from drug overdose among US veterans receiving opioid analgesics: case-cohort study
}

\author{
Tae Woo Park, ${ }^{1}$ Richard Saitz, ${ }^{2}$ Dara Ganoczy, ${ }^{3}$ Mark A Ilgen, ${ }^{34}$ Amy S B Bohnert ${ }^{34}$
}

${ }^{1}$ Departments of Medicine and

Psychiatry and Human

Behavior, The Warren Alpert

Medical School of Brown

University, 111 Plain Street,

Providence, RI 02903.

United States

2Department of Community Health Sciences, Boston

University School of Public

Health, 801 Massachusetts Ave,

Boston, MA 02118, USA

${ }^{3}$ Department of Veterans Affairs, Health Services Research and Development (HSR\&D), 2215

Fuller Road, Ann Arbor, MI

48105, USA

${ }^{4}$ Department of Psychiatry, University of Michigan Medical School, Ann Arbor, MI 48109, USA

Correspondence to: TW Park, tpark1@lifespan.org

Cite this as: BMJ 2015;350:h2698 doi: 10.1136/bmj.h2698

Accepted: 15 April 2015

\section{ABSTRACT}

OBJECTIVE

To study the association between benzodiazepine prescribing patterns including dose, type, and dosing schedule and the risk of death from drug overdose among US veterans receiving opioid analgesics.

DESIGN

Case-cohort study.

SETTING

Veterans Health Administration (VHA), 2004-09.

\section{PARTICIPANTS}

US veterans, primarily male, who received opioid analgesics in 2004-09. All veterans who died from a drug overdose $(n=2400)$ while receiving opioid analgesics and a random sample of veterans $(n=420386)$ who received VHA medical services and opioid analgesics.

\section{MAIN OUTCOME MEASURE}

Death from drug overdose, defined as any intentional, unintentional, or indeterminate death from poisoning caused by any drug, determined by information on cause of death from the National Death Index.

RESULTS

During the study period $27 \%(n=112069)$ of veterans who received opioid analgesics also received benzodiazepines. About half of the deaths from drug overdose ( $n=1185)$ occurred when veterans were concurrently prescribed benzodiazepines and opioids. Risk of death from drug overdose increased with history of benzodiazepine prescription: adjusted hazard ratios were 2.33 (95\% confidence interval 2.05 to 2.64$)$ for former prescriptions versus no prescription and 3.86 (3.49 to 4.26) for current prescriptions versus no prescription. Risk of death from drug overdose

\section{WHAT IS ALREADY KNOWN ON THIS TOPIC}

Benzodiazepines are commonly involved in deaths related to overdose with opioid analgesics, a major cause of injury mortality in the United States

No study has focused specifically on the relation between prescribing patterns of concurrent benzodiazepine and opioid analgesics and death from drug overdose in a large nationwide sample

\section{WHAT THIS STUDY ADDS}

Receipt of benzodiazepines was associated with an increased risk of death from drug overdose among veterans receiving opioid analgesics

The relation between receipt of benzodiazepines and death from drug overdose was dose-dependent: increasing benzodiazepine dose was associated with an increasing risk of death

Temazepam was associated with a decreased risk of death from drug overdose compared with clonazepam

increased as daily benzodiazepine dose increased. Compared with clonazepam, temazepam was associated with a decreased risk of death from drug overdose $(0.63,0.48$ to 0.82$)$. Benzodiazepine dosing schedule was not associated with risk of death from drug overdose.

\section{CONCLUSIONS}

Among veterans receiving opioid analgesics, receipt of benzodiazepines was associated with an increased risk of death from drug overdose in a dose-response fashion.

\section{Introduction}

Deaths from drug overdose, particularly involving opioid analgesics, have increased steadily over the past two decades and are now one of the leading causes of mortality from injury in the United States. ${ }^{1}$ Of the deaths from overdose related to pharmaceuticals, which constituted $58 \%$ of total deaths from drug overdose in 2010, 75\% involved opioid analgesics. ${ }^{2}$ Thirty percent of overdose deaths related to opioid analgesics involved benzodiazepines, drugs commonly prescribed concurrently for patients who receive opioid analgesics. $^{3-5}$ Although the toxicity of benzodiazepines used in isolation is generally considered mild in young and middle aged adults, the risks from oversedation are believed to be magnified when they are combined with other substances with sedating properties, such as opioids. ${ }^{67}$ Also, receipt of benzodiazepines could be a marker of the presence of a severe anxiety disorder, which carries its own risk for death from intentional and unintentional overdose. ${ }^{8} 9$

Trends in deaths from overdose have been connected to patterns of opioid prescribing, an increasingly common treatment for pain. ${ }^{10}$ On a population level, increasing rates of opioid prescribing have been linked to an increased rate of death from overdose ${ }^{11}$; on an individual level, the risk of death from overdose is higher among those receiving higher doses of opioids. $^{12} 13$ One study generated the hypothesis that receipt of benzodiazepines might be associated with increased risk of death from overdose in patients receiving opioid analgesics. ${ }^{13}$ To our knowledge, no large nationwide study has focused on death from drug overdose and receipt of benzodiazepines and their prescribing patterns, such as the daily benzodiazepine dose, the type of benzodiazepine prescribed, or the dosing schedule of the prescription, among those receiving opioids for pain.

We investigated the relation between the receipt of concurrent benzodiazepines and opioid analgesics and death from drug overdose in patients receiving 
prescription opioids for the treatment of acute or chronic pain and pain associated with cancer. Quantification of the magnitude of risk associated with these patterns might help to identify individuals at particularly high risk for death from drug overdose and could potentially inform risk reduction approaches in this population. We hypothesized that receipt of concurrent benzodiazepines and opioid analgesics would be associated with death from drug overdose and that this association would be dose dependent and vary by type of benzodiazepine and dosing schedule. We used a case-cohort study design to examine the relation between history of benzodiazepine prescription, dose, type, and schedule and risk of death from drug overdose over a six year period among a nationwide sample of patients who received treatment with opioid analgesics in the Veterans Health Administration (VHA).

\section{Methods}

\section{Study design and population}

In a case-cohort study design, similar to a nested case-control study, cases and controls are sampled from the same source population. ${ }^{14}$ Controls in the case-cohort design are randomly sampled and, unlike a nested case-control study, matching typically does not occur. Cases and controls in this study were sampled from a source population that consisted of veterans who received Veterans Health Administration (VHA) medical services and opioid analgesic drugs as an outpatient in the financial years 2004-09. The VHA is the largest integrated healthcare system in the US, serving nearly nine million veterans across over 150 hospitals and over 800 community based outpatient centers throughout the US.

We identified 2400 cases in which people died from a drug overdose while they were receiving opioid analgesics. Our control group $(n=420386)$ comprised $5 \%$ annual random samples of the source population during the study period. We attempted to limit the analysis to veterans receiving prescription opioids for the treatment of acute, chronic, and non-terminal cancer pain by excluding 221 veterans prescribed methadone for maintenance purposes and 5816 veterans with indicators of palliative care consultations or hospice care. The final sample size for our study was 422786. To avoid potential immortal time bias, the start of observation for both cases and controls was the date of the first filled prescription for an opioid analgesic after the first medical visit of the year within the study period..$^{15}$ Observation for controls ended either when the veteran died of any cause or at the end of the 2009 financial year, whichever occurred first.

\section{Data sources}

We used data abstracted from the electronic medical records of VHA patients. These included demographic and clinical encounter data from the VHA's National Patient Care Database. Outpatient prescription data were collected from the VHA's Pharmacy Benefits
Management (PBM) Services and consisted of records of filled prescriptions (which included the fill date, days' supply, and schedule). Information on cause of death was obtained from the National Death Index (NDI). ${ }^{16}$ We used two methods to match the NDI for our sample: a full match on the 9 digit social security number and sex and match on at least two of the three parts (day, month, year) of date of birth; or match on at least 7 digits of the social security number plus full match on date of birth, sex, first name, and last name and middle initial when present. More than $99 \%$ of deaths among VHA patients had a full match on social security number.

\section{Deaths from drug overdose}

The NDI reports cause of death using codes from ICD10 (international classification of diseases, 10th revision). Cause of death from drug overdose can be classified as unintentional (X40-45), intentional (X6065), or indeterminate (Y10-15), and specific drugs involved can be listed with a T-code. ${ }^{17}$ Of the 43982 deaths from drug overdose in the US in 2013, 81.1\% were classified as unintentional, $12.4 \%$ intentional, and $6.4 \%$ indeterminate. ${ }^{18}$ Because intent is often difficult to determine, particularly in deaths from poisoning, ${ }^{19}$ and a considerable proportion of death certificates do not specify the $\operatorname{drug}(\mathrm{s})$ involved, ${ }^{2}$ we defined our drug overdose outcome as any intentional, unintentional, or indeterminate overdose death caused by any medication or drug (X40-45, X60-65, Y10-15, without T-code specified).

\section{Drug prescribing patterns}

We examined prescriptions for oral benzodiazepine and opioid analgesics filled on an outpatient basis. As individuals with indications for benzodiazepines might be at a higher risk of death from overdose, ${ }^{8}$ we distinguished periods during which individuals were currently receiving benzodiazepines from periods when they had formerly received them to help deal with unmeasured confounding. ${ }^{20}$ Every person day was classified according to each veteran's history of benzodiazepine prescription, categorized as current, former, or none. "Current” receipt time accumulated starting with a filled benzodiazepine prescription and continued through the end of the prescription, as determined by the days' supply. If another prescription was received and filled, current receipt time continued to accumulate. "Former" receipt time accumulated starting at the end of a current filled benzodiazepine prescription and continued through to the end of the study period, unless another benzodiazepine prescription was filled, at which time it again contributed to current receipt time. Periods before a veteran's first filled prescription for a benzodiazepine or periods for those who never received a benzodiazepine were labeled "none." With regards to each veteran's history of opioid prescription, every person day was classified as "current" or "none." Only days classified as current opioid receipt were included in this study. 
Benzodiazepine types included were alprazolam, chlordiazepoxide, clonazepam, diazepam, lorazepam, temazepam, estazolam, flurazepam, oxazepam, quazepam, and triazolam. The most commonly prescribed benzodiazepines were those on the VHA drug formulary, which consisted of alprazolam, chlordiazepoxide, clonazepam, diazepam, lorazepam, and temazepam. The other benzodiazepines included in the study were prescribed during the study period but at lower rates. Benzodiazepine dose was calculated as diazepam equivalents, according to previously published conversion tables. ${ }^{21}$ Benzodiazepine schedule was classified as regularly scheduled, as needed, or simultaneous regularly scheduled and as needed. Opioid analgesics included in this study were codeine, morphine, oxycodone, hydrocodone, oxymorphone, hydromorphone, fentanyl, propoxyphene, and methadone. We limited methadone prescriptions in this study to those prescribed to treat pain by excluding prescriptions in which dosing instructions indicated the methadone was prescribed for maintenance, oral or effervescent methadone formulations unless the dosing schedule indicated more than once a day dosing, or the dosing schedule was once a day unless the instructions indicated the methadone was prescribed for pain. Buprenorphine is not currently indicated for pain treatment in the VHA and was not included in this analysis. Opioid analgesic dose was calculated as morphine equivalents. ${ }^{2223}$

Both benzodiazepine and opioid analgesic dose were measured with an "as prescribed" approach, which assumes that patients took their benzodiazepines and opioid analgesics according to the prescribers' instructions. ${ }^{24}$ Patients were assumed to be taking the maximum amount described in the prescription, including for "as needed" prescriptions. We calculated dose by adding all diazepam or morphine milligram (mg) equivalents in each prescription and dividing by the number of days' supply. This dose represented the maximum daily dose prescribed and not necessarily the amount actually consumed. If there was an overlap between two prescriptions of the same drug at the same dose and schedule, we assumed that the first prescription was finished before the second prescription was started rather than assuming a higher amount of drug was taken during the overlap. If the overlapping drugs were of a different type, dose, or schedule, than we assumed the second prescription to have started on the date it was filled. Both benzodiazepine and opioid analgesic dose were treated as time varying.

\section{Covariates}

We obtained demographic and diagnostic data from patient records. Demographic data included age, sex, and race. Race was classified as white, black, other, or missing/not recorded. Additionally, each veteran's zip code of residence was linked to US Census data to obtain the percentage of people living below the federal poverty line in that zip code as a proxy measure for socioeconomic status. ${ }^{25}$ Zip code data were missing in
$4.3 \%$ of the sample and were imputed with single imputation. The socioeconomic status variable was categorized into fifths. All diagnoses were made during the year before each veteran's start of observation. Diagnoses were categorized into substance use disorders, post-traumatic stress disorder, other anxiety disorders, depressive disorders, and bipolar or psychotic disorders. Additionally, we measured medical comorbidity using the Charlson comorbidity score. ${ }^{26}$ To assess acuity of mental health and substance use disorder, we also measured the number of related hospital admissions determined by VHA bed section codes in the year before the start of observation. Use of other drugs was assessed by examining the receipt of three categories of drugs in the year before the start of observation: antidepressants, antiepileptic and anti-parkinsonism drugs, and antipsychotic and neuroleptic drugs. These categories were the three most commonly identified classes of pharmaceuticals, after benzodiazepines, that were involved in deaths related to opioid overdose in the US in $2010 .^{2}$

\section{Statistical analysis}

We used $\chi^{2}$ tests to compare baseline patient characteristics between those who received benzodiazepines during the study period and those who did not receive benzodiazepines.

We calculated death rates from overdose according to veterans' history of benzodiazepine prescription, dose, type, and schedule. The denominator for all rates (person years) was adjusted to account for the case-cohort design by multiplying the observation time accumulated for the cohort by the inverse of the sampling fraction. Consequently, all rates are estimates for the entire source population.

We used Cox proportional hazards models to calculate hazard ratios for current and former receipt of benzodiazepines compared with no receipt. All hazard ratios were adjusted for all covariates, including time varying opioid analgesic dose. We used a risk set approach and a robust variance estimator for all multivariable modeling. ${ }^{27}$

Our primary analysis (model 1) studied the association between history of benzodiazepine prescription and death from overdose. In our primary analysis, we tested for an interaction between prescription history and opioid dose. Secondly, we studied the association between benzodiazepine dose, type, and schedule and death from overdose only during times when veterans were currently receiving benzodiazepines and opioid analgesics concurrently (model 2). To determine that any association between opioid dose and death from overdose was not because of confounding by history of benzodiazepine prescription, we examined the association between opioid dose and overdose stratified by prescription history.

We conducted several sensitivity analyses. We examined the association between history of benzodiazepine prescription and death from overdose not known to be intentional by defining a modified overdose death outcome that excluded deaths from overdose coded as 
intentional. We also examined the association between history of benzodiazepine prescription and from overdose with opioid/benzodiazepine by defining a modified overdose death outcome that included only deaths attributed to opioids and/or benzodiazepines (ICD-10 codes X41, X42, X44, X61, X62, X64, Y11, Y12, Y14 in combination with T40.2, T40.3, T40.4, T40.6 or T42.4). We examined the association between history of benzodiazepine prescription and death from overdose in a cohort that excluded patients with a diagnosis of cancer in the year before observation (except for non-melanoma skin cancer). As patients might not take all of their drugs during the days' supply described in the prescription, we expanded the periods during which patients were currently receiving benzodiazepines by $10 \%$ of the days' supply (for example, a 30 day supply would be expanded to 33 days).

Analyses were conducted with SAS version 9.3 (SAS Institute, Cary, NC).

\begin{tabular}{|c|c|c|}
\hline \multirow[b]{2}{*}{ Characteristic } & \multicolumn{2}{|c|}{ Receipt of benzodiazepine $^{*}$} \\
\hline & No $(n=310717)$ & Yes $(n=112069)$ \\
\hline Male & $291070(94)$ & $102252(91)$ \\
\hline \multicolumn{3}{|l|}{ Age (years): } \\
\hline $18-29$ & $9256(3)$ & $2639(2)$ \\
\hline $30-39$ & $15053(5)$ & $5791(5)$ \\
\hline $40-49$ & $35961(12)$ & $15841(14)$ \\
\hline $50-59$ & $83772(27)$ & 38471 (34) \\
\hline $60-69$ & $77147(25)$ & $25366(23)$ \\
\hline$\geq 70$ & $89528(29)$ & $23961(21)$ \\
\hline \multicolumn{3}{|l|}{ Race: } \\
\hline Black & $57286(18)$ & $12600(11)$ \\
\hline White & $217663(70)$ & $89764(80)$ \\
\hline Other/missing & $35768(12)$ & $9705(9)$ \\
\hline Hispanic & $14285(5)$ & $5470(5)$ \\
\hline \multicolumn{3}{|l|}{ Area level poverty (fifths): } \\
\hline 1 (wealthiest) & $61992(20)$ & $22565(20)$ \\
\hline 2 & $61741(20)$ & $22853(20)$ \\
\hline 3 & $61377(20)$ & $23140(21)$ \\
\hline 4 & $61465(20)$ & $23113(21)$ \\
\hline 5 (poorest) & $64142(21)$ & $20398(18)$ \\
\hline Substance use disorder (SUD)† & $29544(10)$ & $14712(13)$ \\
\hline $\begin{array}{l}\text { Recent admission to hospital related to } \\
\text { mental health or SUDt }\end{array}$ & $4607(1)$ & $4798(4)$ \\
\hline Post-traumatic stress disordert & $27995(9)$ & $24759(22)$ \\
\hline Other anxiety disordert & $15266(5)$ & $22369(20)$ \\
\hline Depressiont & 58627 (19) & $42827(38)$ \\
\hline Bipolar/psychotic disordert & $13587(4)$ & 11795 (11) \\
\hline Cancert & $73929(24)$ & $25821(23)$ \\
\hline \multicolumn{3}{|l|}{ Charlson comorbidity indext: } \\
\hline 0 & $129358(42)$ & $44003(39)$ \\
\hline 1 & $73260(24)$ & $27448(24)$ \\
\hline$\geq 2$ & $108099(35)$ & $40618(36)$ \\
\hline \multicolumn{3}{|l|}{ Receipt of other drugst: } \\
\hline Antidepressants & $96557(31)$ & $65547(58)$ \\
\hline Antiepileptic and anti-parkinsonism drugs & $72630(23)$ & $43063(38)$ \\
\hline Antipsychotic and neuroleptic drugs & 32734 (11) & $27817(25)$ \\
\hline
\end{tabular}

${ }^{*} \mathrm{P}<0.001$ for all comparisons between groups.

tAll conditions and events measured in year up to and including day of first filled prescription for opioid during observation period and are not mutually exclusive.

\section{Patient involvement}

There was no direct patient involvement in this study. The VHA datasets used for analysis did not include names and social security numbers of patients.

\section{Results}

Sample

Of the 422786 veterans in the study population, 112069 (27\%) had filled at least one prescription for benzodiazepine during the study period (table 1). Those who received benzodiazepines were more likely to be women (33\% of women $v 26 \%$ of men received benzodiazepines), middle aged, white, and live in wealthier areas. Additionally, they were more likely to have had a recent hospital admission for mental health or substance use disorder, to have a diagnosis of a substance use disorder or several psychiatric disorders, including post-traumatic stress disorder, other anxiety disorders, depression, and bipolar or psychotic disorders, and to use other drugs.

\section{Unadjusted rates of death from drug overdose}

Of the 2400 veterans in the study population who died from a drug overdose while receiving opioid analgesics, 1185 (49\%) died during a period in which they had been prescribed concurrent benzodiazepines (table 2). Unadjusted rates of death from drug overdose were higher during periods when veterans currently received benzodiazepines than in those when veterans formerly received them or had not received them at all. Unadjusted rates of death from overdose increased at consecutively higher categories of daily benzodiazepine dose. Periods during which veterans received lorazepam and temazepam had lower rates of death from overdose than periods involving the other benzodiazepine types. Overdose death rates were higher in periods in which benzodiazepines were prescribed as regularly scheduled and as needed drugs compared with the other schedules. When the rates of death from overdose were stratified by categories of daily opioid dose (figure), the difference in rates between periods of current and former benzodiazepine receipt was not significant at the lowest opioid dose category but was significant at higher opioid dose categories.

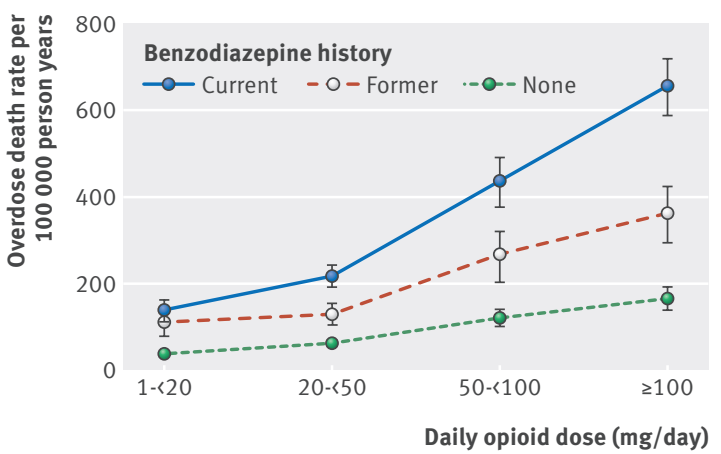

Unadjusted death rates for drug overdose by benzodiazepine prescription history and daily opioid dose. Error bars represent $95 \%$ confidence intervals. Unadjusted overdose death rates are estimates for entire source population 


\section{Adjusted analyses}

Compared with periods in which veterans had not received benzodiazepines, periods of current benzodiazepine receipt (hazard ratio 3.86, 95\% confidence interval 3.49 to 4.26) and former benzodiazepine receipt (2.33, 2.05 to 2.64$)$ were associated with an increased risk of death from overdose (table 3 ). The interaction between benzodiazepine history and opioid dose was not significant $(\mathrm{P}=0.60)$, indicating that the relations between benzodiazepine prescription and opioid dose and death from overdose were neither greater nor less than additive. Categories of higher benzodiazepine doses were associated with greater risk of overdose death. Temazapam $(0.63,0.48$ to 0.82$)$ was associated with lower risk of death from overdose compared with the most commonly prescribed benzodiazepine, clonazepam. All other benzodiazepines had similar risk compared with clonazepam. After adjustment, we did not find an association between benzodiazepine dosing schedule and death from overdose. After stratification by history of benzodiazepine prescription, compared with the lowest opioid dose category, higher opioid dose categories were associated with increasingly greater risk of death from overdose during periods of no benzodiazepine receipt, former benzodiazepine receipt, and current benzodiazepine receipt (table 4).

\section{Sensitivity analyses}

We conducted several sensitivity analyses to test the robustness of the results of our primary analysis. We found that the exclusion of deaths from intentional overdose, deaths from overdose not related to opioids or

\begin{tabular}{|c|c|c|c|}
\hline & $\begin{array}{l}\text { No of deaths } \\
\text { from overdose }\end{array}$ & $\begin{array}{l}\text { Person } \\
\text { years }\end{array}$ & $\begin{array}{l}\text { Overdose death rate } \\
\text { per } 100000 \text { person } \\
\text { years }(95 \% \mathrm{Cl})^{\star}\end{array}$ \\
\hline \multicolumn{4}{|c|}{ Benzodiazepine prescription history: } \\
\hline None & 794 & 1116346 & $71(66$ to 76$)$ \\
\hline Former & 421 & 225171 & $187(170$ to 205$)$ \\
\hline Current & 1185 & 375332 & 316 (298 to 334) \\
\hline \multicolumn{4}{|c|}{ Daily benzodiazepine dose (mg/day): } \\
\hline 0 & 421 & 376645 & 112 (101 to 123$)$ \\
\hline$>0-10$ & 241 & 158676 & 152 (133 to 172$)$ \\
\hline$>10-20$ & 366 & 121411 & 301 (271 to 333) \\
\hline$>20-30$ & 237 & 45718 & 518 (454 to 586) \\
\hline$>30-40$ & 169 & 28170 & $600(513$ to 694$)$ \\
\hline$>40$ & 172 & 21357 & 805 (689 to 930$)$ \\
\hline \multicolumn{4}{|l|}{ Benzodiazepine type: } \\
\hline Clonazepam & 288 & 76017 & 379 (336 to 424) \\
\hline Diazepam & 276 & 72444 & 381 (337 to 427) \\
\hline Alprazolam & 182 & 63155 & 288 (248 to 332) \\
\hline Lorazepam & 110 & 64587 & 170 (140 to 204) \\
\hline Temazepam & 88 & 57301 & $154(123$ to 187$)$ \\
\hline Other & 20 & 6464 & 309 (189 to 459) \\
\hline Multiple & 221 & 35365 & 625 (545 to 710$)$ \\
\hline \multicolumn{4}{|l|}{ Benzodiazepine schedule: } \\
\hline Regularly scheduled only & 449 & 169515 & 265 (241 to 290) \\
\hline As needed only & 638 & 190933 & 334 (309 to 361) \\
\hline Both & 98 & 14884 & 658 (535 to 795$)$ \\
\hline
\end{tabular}

benzodiazepines, people with cancer, and an expansion of the periods during which patients were currently prescribed benzodiazepines resulted in only minor differences in the degree of association between history of benzodiazepine prescription and death from overdose, and no substantive differences in the inferences drawn from analyses.

\section{Discussion}

Although we were unable to determine whether benzodiazepine prescribing patterns are the direct cause of death from drug overdose because of the observational design of this study, we found that receipt of concurrent benzodiazepines was associated with an increased risk of death from overdose in this large national sample of veterans who received opioid analgesics. Notably, about half of the deaths from overdose occurred while veterans were receiving concurrent benzodiazepines and opioids. Benzodiazepine dose was also found to be associated with an increased risk of death from overdose in a dose-response fashion. Compared with clonazepam, temazepam was associated with a decreased risk of death from overdose. Current receipt of benzodiazepines was associated with a greater magnitude of risk than former receipt. These associations remained significant in analyses adjusted for potential confounders.

Table 3 | Adjusted hazard ratios for deaths from drug overdose by history of benzodiazepine prescription, daily benzodiazepine dose, benzodiazepine type, and benzodiazepine schedule

Hazard ratio $(95 \% \mathrm{Cl})$

Model 1*

\begin{tabular}{|c|c|}
\hline \multicolumn{2}{|c|}{ Benzodiazepine prescription history: } \\
\hline None & 1.00 (reference) \\
\hline Former & $2.33(2.05$ to 2.64$)$ \\
\hline Current & 3.86 (3.49 to 4.26$)$ \\
\hline \multicolumn{2}{|l|}{ Model 2† } \\
\hline \multicolumn{2}{|c|}{ Daily benzodiazepine dose (mg/day): } \\
\hline$>0-10$ & 1.00 (reference) \\
\hline$>10-20$ & $1.69(1.42$ to 2.01$)$ \\
\hline$>20-30$ & $2.34(1.91$ to 2.86$)$ \\
\hline$>30-40$ & 2.65 (2.10 to 3.33$)$ \\
\hline$>40$ & 3.06 (2.38 to 3.92) \\
\hline \multicolumn{2}{|l|}{ Benzodiazepine type: } \\
\hline Clonazepam & 1.00 (reference) \\
\hline Alprazolam & $0.93(0.75$ to 1.14$)$ \\
\hline Diazepam & 0.93 (0.77 to 1.13$)$ \\
\hline Lorazepam & $0.79(0.62$ to 1.00$)$ \\
\hline Temazepam & $0.63(0.48$ to 0.82$)$ \\
\hline Other & 1.09 (0.67 to 1.77$)$ \\
\hline Multiple & 1.05 (0.83 to 1.33$)$ \\
\hline \multicolumn{2}{|l|}{ Benzodiazepine schedule: } \\
\hline Regularly scheduled & 1.00 (reference) \\
\hline As needed only & 0.98 (0.86 to 1.13$)$ \\
\hline Both & 1.07 (0.80 to 1.43$)$ \\
\hline
\end{tabular}

*Adjusted for sex, age, race, ethnicity, area level poverty, time varying daily opioid dose, recent admission to hospital related to SUD, Charlson comorbidity index, diagnosis of substance use disorder, post-traumatic stress disorder, other anxiety disorder, depression, bipolar/psychotic disorder, and cancer, and use of other drugs.

tAdjusted as for model 1 but included only periods when veterans were currently receiving benzodiazepines. 


\begin{tabular}{|c|c|}
\hline & Hazard ratio $(95 \% \mathrm{CI})$ \\
\hline \multicolumn{2}{|c|}{ No receipt of benzodiazepine } \\
\hline \multicolumn{2}{|c|}{ Daily opioid dose (mg/day): } \\
\hline$>0-<20$ & 1.00 (reference) \\
\hline $20-<50$ & 1.57 (1.28 to 1.91$)$ \\
\hline $50-<100$ & $2.81(2.26$ to 3.50$)$ \\
\hline$\geq 100$ & $3.30(2.62$ to 4.16$)$ \\
\hline \multicolumn{2}{|c|}{ Former receipt of benzodiazepine } \\
\hline \multicolumn{2}{|c|}{ Daily opioid dose (mg/day): } \\
\hline$>0-<20$ & 1.00 (reference) \\
\hline $20-<50$ & $1.17(0.86$ to 1.60$)$ \\
\hline $50-<100$ & 2.33 (1.69 to 3.21$)$ \\
\hline$\geq 100$ & $3.00(2.19$ to 4.11$)$ \\
\hline \multicolumn{2}{|c|}{ Current receipt of benzodiazepine } \\
\hline \multicolumn{2}{|c|}{ Daily opioid dose (mg/day): } \\
\hline$>0-<20$ & 1.00 (reference) \\
\hline $20-<50$ & 1.54 (1.24 to 1.90$)$ \\
\hline $50-<100$ & 2.95 (2.37 to 3.66$)$ \\
\hline$\geq 100$ & $3.92(3.17$ to 4.85$)$ \\
\hline \multicolumn{2}{|c|}{$\begin{array}{l}\text { *Separate models created for each daily opioid dose category adjusted } \\
\text { for sex, age, race, ethnicity, area level poverty, time varying daily opioid } \\
\text { dose, recent admission to hospital related to substance use disorder, } \\
\text { Charlson comorbidity index, diagnosis of substance use disorder, } \\
\text { post-traumatic stress disorder, other anxiety disorder, depression, } \\
\text { bipolar/psychotic disorder, and cancer, and use of other drugs. }\end{array}$} \\
\hline
\end{tabular}

\section{Strengths and weaknesses of study}

The strengths of this study include the large size of the study cohort and the ability to study a national sample of patients from the largest health system in the US. Additionally, we were able to adjust for a range of potential confounders, including demographic, socioeconomic, and medical and mental health diagnostic data. Although we studied a large national cohort, all study participants were US Veterans and most were men; both of which are characteristics associated with accidental poisoning mortality. ${ }^{28}$ This could limit the generalizability of the study. Because we examined only treatment received within the VHA in this study, we might not have captured encounters that could affect risk of death from overdose, such as drugs received from non-VHA prescribers. Additionally, as our daily dose calculations for benzodiazepines and opioid analgesics assume that veterans take drugs exactly as prescribed, we might not have accurately measured the amount of drugs taken on any given day. Veterans might have retained drugs beyond the period for which they were prescribed or received additional drugs from non-VHA prescribers and subsequently took larger doses than indicated by the prescribers' instructions. If this behavior is associated with receipt of benzodiazepines and with death from overdose, then our effect estimates might have been inflated. Because VHA pharmacy data are not linked to indications or diagnoses, we were unable to ascertain the indications for which the benzodiazepines were prescribed. Thus, we are unable to know if the association between benzo- diazepine prescription and death from overdose differs by indication or draw inferences to patients with specific indications.

\section{Findings in context}

The findings of this study were largely consistent with studies that have suggested that there are risks associated with concurrent benzodiazepine and opioid use. Dunn and colleagues found an association between receipt of sedative-hypnotic drugs and increased risk of combined fatal and non-fatal overdoses. ${ }^{13}$ Other studies have looked at smaller subgroups of patients taking opioid analgesics. In a study limited to patients with severe respiratory disease, receipt of concurrent benzodiazepines and opioid analgesics was associated with increased risk of total mortality. ${ }^{29}$ In a study of veterans with post-traumatic stress disorder, receipt of concurrent benzodiazepines and opioid analgesics, combined with antidepressants, was found to be associated with increased risk of adverse events. ${ }^{30}$ Our study extends this literature in several ways. We studied a much larger nationwide sample focusing exclusively on benzodiazepine prescribing patterns and their association with fatal overdoses. We found a dose-dependent relation between benzodiazepine dose and death from overdose and were also able to more rigorously deal with issues of confounding in our analysis because of the larger sample size.

Because benzodiazepines were more likely to be prescribed to those with substance misuse and other psychiatric disorders, conditions that carry their own risk for death from overdose, ${ }^{8}$ the association between receipt of benzodiazepines and death from overdose might be partially explained by these underlying conditions or the severity of those conditions. ${ }^{31}$ We attempted to examine this possibility in two ways. We adjusted for baseline characteristics of patients, including demographic information, medical and mental health diagnoses, and the daily opioid dose, in a multivariable model. Additionally, by distinguishing between periods of current and former receipt of benzodiazepines, we dealt with some unmeasured confounding. Nonetheless, because those with current receipt of benzodiazepines might have had more severe conditions for which benzodiazepines were prescribed than those with former receipt, some residual confounding could exist. Patients with more severe addiction or more severe psychiatric morbidity could be more likely to take any drugs in greater quantities than prescribed, as well as illicit drugs, leading to an increased risk of death from overdose. Additionally, it is unclear through our analysis the degree to which benzodiazepines contributed to the actual cause of death from overdose. Half of such deaths occurred during periods when benzodiazepines were not prescribed, and, although the risk of death from overdose increased in a benzodiazepine dose-response fashion, this could reflect an effect of greater psychiatric severity, or other differences between patients who did and did not receive benzodiazepines, rather than the benzodiazepine itself. Thus it is 
important to note that within the present study, benzodiazepines might be better conceptualized as a marker of risk with unknown direct causal links to death from overdose.

The risk of death from overdose was increased during periods of former benzodiazepine receipt. In addition to having a greater risk of death from overdose because of having the underlying conditions for which benzodiazepines are prescribed, the increased risk in those formerly prescribed benzodiazepines could be explained by continued use of benzodiazepines obtained illicitly or through non-VHA providers. Although we found that expanding the period in which veterans were currently being prescribed benzodiazepines had a negligible impact on risk of death from overdose, those formerly prescribed benzodiazepines might still have had leftover benzodiazepines that they continued to use well past the end date of their prescription.

Benzodiazepines are often prescribed for patients who also receive high doses of opioid analgesics. ${ }^{4} 5$ Studies across several clinical samples and with varying methods (including the present sample) have now replicated a finding of an association between opioid dose and risk of death from overdose. ${ }^{12} 1332$ Our findings further show that this association is not caused by confounding by history of benzodiazepine prescription or by several control covariates we included here that were not included in the previous study in this sample. ${ }^{12}$ The association between opioid dose and death from overdose did not seem to differ by history of benzodiazepine prescription.

Although receipt of benzodiazepines and the benzodiazepine dose were associated with increased risk of death from overdose, the formulation and dosing schedule were found to be largely unrelated after adjustment for potential confounders. Temazepam ${ }^{33}$ and alprazolam ${ }^{34}$ have been associated with increased toxicity compared with other benzodiazepines in studies of deliberate self poisoning with benzodiazepines. In contrast, we found that, when compared with clonazepam, temazepam was associated with a decreased risk of death from overdose. Possible explanations are the pharmacokinetics of the drugs and selection factors related to the use of temazepam, but differentiation between these two was beyond the scope of the present study. More research is needed to understand why temazepam was associated with a lower risk of death from overdose. Although there is limited evidence that an as needed benzodiazepine dosing schedule could pose risks for patients, particularly in elderly people and those with substance use disorders, ${ }^{35} 36$ we found no association between as needed dosing and risk of death from overdose compared with regularly scheduled dosing after adjustment for potential confounders.

Although the design of this study does not allow for the determination of the extent to which benzodiazepines cause deaths from overdose, it does indicate a need for clinicians to be aware of the increased risk among patients currently receiving benzodiazepines and opioids and that the risk might be higher among those receiving higher doses of either or both drugs. This risk might be due to risks inherent to those prescribed benzodiazepines, such as the presence of an anxiety condition and/or to the benzodiazepine itself. Thus, clinicians should be cautious when prescribing benzodiazepines to this group. Although it is unknown whether patients with comorbid pain and anxiety who use opioids and benzodiazepines would benefit from the same interventions to reduce risk of death from overdose as patients using opioids alone, clinicians might consider including this group in overdose prevention efforts, such as naloxone training for caregivers. ${ }^{37}$

Contributors: All authors have fulfilled authorship criteria per ICMJE guidelines and have approved the manuscript and this submission. TP, $\mathrm{RS}$, and ASBB were involved in the conception or design of the work. TP, DG, and ASBB were involved in acquisition and analysis of data. All authors were involved in interpretation of data. TP and ASBB drafted the manuscript and all authors revised the work critically for important intellectual content. All coauthors approve this version of the manuscript and agree to be accountable for all aspects of the work in ensuring that questions related to the accuracy or integrity of any part of the work are appropriately investigated and resolved. TP is guarantor.

Funding: This work was supported by funding from a Veteran Affairs Health Services Research and Development Service Career Development Award (CDA-09-204; ASBB.) and by the National Institutes of Health (R03 AG042899). The sponsors had no role in the design and conduct of the study; in the collection, analysis, and interpretation of the data; or in the preparation, review, or approval of the manuscript of the decision to submit.

Competing interests: All authors have completed the ICMJE uniform disclosure form at www.icmje.org/coi_disclosure.pdf and declare: financial support as specified above; RS has received consultant and annual travel fees from The BMJ as editor of the journal Evidence Based Medicine; no other relationships or activities that could appear to have influenced the submitted work.

Ethical approval: The study procedures received approval from the Ann Arbor VA human studies committee, which waived the requirement for informed consent.

Transparency declaration: All authors affirm that this manuscript is an honest, accurate, and transparent account of the study being reported; that no important aspects of the study have been omitted; and that any discrepancies from the study as planned (and, if relevant, registered) have been explained.

Data sharing: Veterans Health Administration (VHA) treatment data are maintained and available for the VHA community through VA Informatics and Computing Infrastructure (www.hsrd.research.va.gov/ for_researchers/vinci/).

This is an Open Access article distributed in accordance with the Creative Commons Attribution Non Commercial (CC BY-NC 4.0) license, which permits others to distribute, remix, adapt, build upon this work non-commercially, and license their derivative works on different terms, provided the original work is properly cited and the use is non-commercial. See: http://creativecommons.org/licenses/ by-nc/4.0/.

Paulozzi LJ. Prescription drug overdoses: a review. I Safety Res 2012;43:283-9.

2 Jones CM, Mack KA, Paulozzi LJ. Pharmaceutical overdose deaths, United States, 2010. JAMA 2013;309:657-9.

3 Hermos JA, Young MM, Gagnon DR, Fiore LD. Characterizations of long-term oxycodone/acetaminophen prescriptions in veteran patients. Arch Intern Med 2004;164:2361-6.

4 Morasco BJ, Duckart JP, Carr TP, Deyo RA, Dobscha SK. Clinical characteristics of veterans prescribed high doses of opioid medications for chronic non-cancer pain. Pain 2010;151:625-32.

5 Saunders KW, Von Korff M, Campbell Cl, et al. Concurrent use of alcohol and sedatives among persons prescribed chronic opioid therapy: prevalence and risk factors. J Pain 2012;13:266-75.

6 Salzman C. The APA task force report on benzodiazepine dependence, toxicity, and abuse. Am J Psychiatry 1991;148:151-2.

7 Cone EJ, Fant RV, Rohay JM, et al. Oxycodone involvement in drug abuse deaths. II. Evidence for toxic multiple drug-drug interactions. J Anal Toxicol 2004:28:616-24. 
8 Bohnert AS, Ilgen MA, Ignacio RV, McCarthy JF, Valenstein M, Blow FC. Risk of death from accidental overdose associated with psychiatric and substance use disorders. Am J Psychiatry 2012;169:64-70.

9 Pfeiffer PN, Ganoczy D, Ilgen MA, et al. Comorbid anxiety as a suicide risk factor among depressed veterans. Depress Anxiety 2009;26:752-7.

10 Okie S. A flood of opioids, a rising tide of deaths. N Engl J Med 2010;363:1981-5.

11 Paulozzi LI, Budnitz DS, Xi Y. Increasing deaths from opioid analgesics in the United States. Pharmacoepidemiol Drug Saf 2006; 15:618-27.

12 Bohnert AS, Valenstein M, Bair MJ, et al. Association between opioid prescribing patterns and opioid overdose-related deaths. JAMA 2011;305:1315-21

13 Dunn KM, Saunders KW, Rutter CM, et al. Opioid prescriptions for chronic pain and overdose: a cohort study. Ann Intern Med 2010;152:85-92.

14 Prentice RL. A case-cohort design for epidemiologic cohort studies and disease prevention trials. Biometrika 1986;73:1-11.

15 Suissa S. Immortal time bias in pharmaco-epidemiology. Am J Epidemiol 2008;167:492-9.

16 Centers for Disease Control and Prevention. National death index. www.cdc.gov/nchs/ndi.htm.

17 Wysowski DK. Surveillance of prescription drug-related mortality using death certificate data. Drug Saf 2007;30:533-40.

18 Centers for Disease Control and Prevention. National vital statistics system mortality data. www.cdc.gov/nchs/deaths.htm.

19 Lindqvist P, Gustafsson L. Suicide classification-clues and their use. A study of 122 cases of suicide and undetermined manner of death. Forensic Sci Int 2002;128:136-40.

20 Arbogast PG, Ray WA. Use of disease risk scores in pharmacoepidemiologic studies. Stat Methods Med Res 2009;18:67-80.

21 Galanter M, Kleber H, ed. Textbook of substance abuse. 4th ed. American Psychiatric Publishing, 2008

22 Gammaitoni AR, Fine P, Alvarez N, McPherson ML, Bergmark S. Clinical application of opioid equianalgesic data. Clin J Pain 2003;19:286-97.

23 Prommer E. Oxymorphone: a review. Support Care Cancer 2006;14:109-115.

24 Valenstein M, Taylor KK, Austin K, Kales HC, McCarthy JF, Blow FC. Benzodiazepine use among depressed patients treated in mental health settings. Am J Psychiatry 2004;161(4):654-661.
25 Krieger N, Waterman PD, Chen JT, Rehkopf DH, Subramanian SV. Geocoding and monitoring US socioeconomic inequalities in health: an introduction to using area-based socioeconomic measures-the public health disparities geocoding project monograph. www.hsph. harvard.edu/thegeocodingproject/.

26 Quan $\mathrm{H}$, Sundararajan V, Halfon P et al. Coding algorithms for definin comorbidities in ICD-9-CM and ICD-10 administrative data. Med Care 2005; $43: 1130-9$

27 Langholz B, Jiao J. Computational methods for case-cohort studies. Comput Stat Data Anal 2007:51:3737-48

28 Bohnert ASB, Ilgen MA, Galeo S, et al. Accidental poisoning mortality among patients in the Department of Veteran Affairs health system. Med Care 2011:49:393-6.

29 Ekstrom MP, Bornefalk-Hermansson A, Abernethy AP, Currow DC Safety of benzodiazepines and opioids in very severe respiratory disease: national prospective study. BMJ 2014;348:g445.

30 Hawkins El, Malte CA, Grossbard I, Saxon AJ, Imel ZE, Kivlahan DR. Comparative safety of benzodiazepines and opioids among veterans affairs patients with posttraumatic stress disorder. J Addict Med 2013;7:354-62.

31 Salas M, Hofman A, Stricker BH. Confounding by indication: An example of variation in the use of epidemiologic terminology. Am J Epidemiol 1999;149:981-3.

32 Gomes T, Mamdani MM, Dhalla IA, Paterson JM, Juurlink DN. Opioid dose and drug-related mortality in patients with nonmalignant pain. JAMA Intern Med 2011;171:686-91.

33 Buckley NA, Dawson AH, Whyte IM, O'Connell DL. Relative toxicity of benzodiazepines in overdose. BMJ 1995;310:21-21.

34 Isbister GK, O'Regan L, Sibbritt D, Whyte IM. Alprazolam is relatively more toxic than other benzodiazepines in overdose. Br J Clin Pharmacol 2004:58:88-95.

35 Sgadari A, Lapane KL, Mor V, Landi F, Bernabei R, Gambassi G. Oxidative and nonoxidative benzodiazepines and the risk of femur fracture. The Systematic Assessment of Geriatric Drug Use Via Epidemiology Study Group. J Clin Psychopharmacol 2000;20:234-9.

36 Center for Substance Abuse Treatment. TIP 43: medication-assisted treatment for opioid addiction in opioid treatment programs. Center for Substance Abuse Treatment, 2005. DHHS Publication No (SMA) 05-4048

37 Beletsky L, Rich JD, Walley AY. Prevention of fatal opioid overdose. IAMA 2012;308:1863-4.

(C) BMJ Publishing Group Ltd 2015 